\title{
EXPERIMENTAL ANALYSIS OF EXHAUST NOISE USING A MUFFLER AND A PARTICULATE TRAP IN A DIESEL ENGINE
}

\author{
R. Sundara Raman ${ }^{1, *}$, G. Sankara Narayanan², N. Manoharan ${ }^{3}$ \\ and S. Sendilvelan ${ }^{4}$ \\ ${ }^{1}$ Department of Mechanical Engineering, AMET University, 135, East Coast Road, \\ Chennai-603 112 \\ ${ }^{2}$ PG Studies \& Research, Sree Sastha Institute of Engineering and Technology, \\ Chembarambakkam, Chennai. \\ ${ }^{3}$ AMET University, Chennai. \\ ${ }^{4}$ Department of Mechanical Engineering, Dr. MGR Educational and Research Institute \\ University, Maduravoyal, Chennai. \\ *E-mail: sundararaman792@gmail.com
}

\begin{abstract}
The control of the engine exhaust noise by a particulate trap for a diesel engine is investigated. Adequate theoretical background about the sound waves and engine noise is provided with a brief discussion on the sources of engine noise, exhaust noise generation and the principle of suppression. The experimental work comprises of design and development of the particulate trap for the given engine to control the exhaust noise level. The noise level reduction is also studied using a muffler. The reduction in noise level using particulate trap and muffler is experimentally investigated and compared.
\end{abstract}

Keywords: Engine Noise, Noise Level, Particulate Trap, Muffler.

(C) RASĀYAN. All rights reserved

\section{INTRODUCTION}

The pressure in an exhaust pipe system is of pulsating nature and it was also investigated into the best way of how these pulsations can be controlled so that they are of maximum advantage to the engine performance. The methods and conclusions differ on a number of points. Pressure fluctuations in a conventional exhaust manifold generally impair engine performance by interfering with the proper exhaust and charging of the engine cylinder. Exhaust energy has become a drag on the engine and a spoiler of performance. At full load, the energy lost in this way would be the same order as heat equivalent of the indicated horsepower. This energy is not only exhibited as high exhaust temperatures but also in the form of pressure pulsation or waves travelling in the form of sound along the exhaust system providing acceleration and retardation of the gas particles as they pass along the exhaust system. About $10 \%$ of the energy of the fuel is contained in the pulsations produced in the exhaust system of compression ignition engines ${ }^{1}$.

\section{Literature Survey}

Keisuke Yoshimori et al. ${ }^{2}$ have designed a new type of exhaust silencer made of porous sintered aluminium. They analysed the noise abatement using fluid dynamic analysis technique and concluded that noise reduction results mainly from the fluid effects arising from the gas permeability of the material, after considering the effects of flattening of the velocity profile, heat dispersion effect, decrease in turbulence of flow and smoothing of exhaust pulsation ${ }^{2}$.

Atsuo shimura ${ }^{3}$ has described the longitudinal vibration model as a beam and applied to the high frequency vibration that causes the noise radiated from the exhaust system. Some methods of reducing such noise radiation by isolating the vibration from the front exhaust pipe were also studied. The 
described methods are adding mass to the front pipe, change in the properties of pipe material of smaller Young's modulus, installing flexible pipes composed of two sections and so on. ${ }^{3}$

Peter et $\mathrm{l}^{4}$ have discussed about the Helmholtz resonator, which has been used in industry for a long time to reduce the noise from the exhaust system in vehicle or machinery. They have developed a thin rectangular shell type muffler element which can be effectively utilized for reducing exhaust noise and proposed a point impedance method to solve for natural frequencies and mode shapes of the above combined system ${ }^{4}$.

Robert $e t a l^{5}$ reformulated the acoustical finite element to make geometric parameters, explicit variable of the matrix equation. They also included several different illustrations of acoustical design that depends upon geometric shape, using new formulation; the synthesis of an appropriate shape to get a desired response, the computation of shape sensitivity and automated shape optimization or minimization of a given objective function. ${ }^{5}$

Fransisco et $a l^{6}$ generated a tool for sound attenuation properties for perforated ducts and influence on attenuation by different arrangements of perforated tubes related to back pressure development by the muffler. They have validated the results obtained in a particularly simple case and confirmed the feasibility of the proposed methodology ${ }^{6}$.

Douglas $e t a l^{7}$ developed a model to predict the reduction of emission levels using the oxidation catalysts systems for two-stroke engines and explained the thermodynamic and gas dynamics aspects and the limitation of the models. They have recommended for the evaluation of a catalyst simulation suitable for use within a full engine model. ${ }^{7}$ They have also studied about the standard exhaust noise for common engines which is given in Table-1.

Table-1: Standard Exhaust Noise for Common Engines ${ }^{2}$

\begin{tabular}{l|c|c}
\hline \multicolumn{1}{c|}{ Engine Type } & $\begin{array}{c}\text { Standard Db (A) } \\
\text { At a distance of } 0.5 \mathrm{~m}\end{array}$ & RPM \\
\hline Motor Boat Engine & 130 & 3000 \\
\hline $\begin{array}{l}\text { Single cylinder } \\
\text { Engine for motor cycle }\end{array}$ & 85 & 1500 \\
\hline Twin cylinder diesel engine & 95 & 1500 \\
\hline Three cylinder diesel engine & 110 & 1500 \\
\hline Four cylinder diesel engine & 120 & 1500 \\
\hline
\end{tabular}

\section{Sound Waves}

The phenomenon of transmission of elastic wave can be explained by the following examples. Let us consider a coiled spring in free position. In free position the elasticity of the springs keeps the coils evenly spaced. ${ }^{2}$ Now let a sudden compressive movement is imparted to one end of the spring. The spring is compressed and energy is stored. The resulting momentum displaces coils to their original position. The wave is propagated towards the other end along the spring. ${ }^{3}$

The to and fro motion of the piston causes the adjacent air to compress and this motion is carried along the tube due to the elasticity of the medium present in the tube. A sinusoidal wave is generated whose frequency is same as that of the piston and amplitude is determined by the displacement of the piston. ${ }^{3}$ The time taken by the wave to travel a certain distance depends upon the elasticity and the density of the medium. It is found that for air the velocity of sound propagation is given by:

$$
\mathrm{C}^{3}=\sqrt{ } 4 \mathrm{P} / \rho
$$

Where, $\mathrm{C}=$ sonic velocity in $\mathrm{m} / \mathrm{s} ; \mathrm{P}=$ Atmospheric pressure in bar; $\rho=$ Density in $\mathrm{kg} /$ cubic meters.

\section{Combustion Noise}

If more fuel is present in the cylinder at the end of delay period and undergoes rapid combustion when 
ignition sets in, the rate of pressure rise and the peak pressure attained will be greater. ${ }^{3}$ During this combustion the piston is around top dead centre and is almost stand still. Too rapid a pressure rise and severe pressure impulse at this position of the piston will result in combustion noise called Diesel Knock. The severity of the knock reactions is in proportion to the mass enflamed. ${ }^{3}$ The regions of premixed flame are probably hotter than the regions where liquid droplets are present. As such, the knock reaction may be propagated mainly in the low luminosity stage of the flame. ${ }^{4}$

\section{Origin of Exhaust Noise}

The gas in an engine cylinder is under considerably high pressure and temperature at the end of firing stroke. ${ }^{4}$ When the exhaust valve opens the gas is forcibly ejected out of the cylinder into the exhaust system. The gases have the mass and elasticity. When the gases are let out in the open air without any exhaust processing, the noise after travelling a short distance assumes the form of a detonation like pulse with steep wave fronts. These acoustic shock waves are unendurable even for a short time, since they possess very high intensities. ${ }^{5}$

\section{Phenomenon of Exhaust Noise}

When the gases are released and again the exhaust valve is closed the gas over- runs, i.e., stretches itself out well beyond its normal length. ${ }^{6}$ This coupled with the cooling of the gas and condensing of the vapour present in the gas results in a partial vacuum followed by a slug. Thus there is a severe oscillating condition setup in the exhaust conduit. Pressure waves of considerable amplitude are reflected back and forth in the pipeline at acoustic velocities. ${ }^{7}$ The frequency of the reflected wave depends primarily upon the pipe length and somewhat on pipe diameter and gas temperature. The acoustical exhaust boom is caused by the synchronization of the explosion frequency in the engine with the natural frequency of the gas in the exhaust system acting as an open-ended organ pipe. ${ }^{7}$ In practice it is usual that the higher harmonics of the system coincide with either fundamental firing rate or a harmonic of the fundamental.

The noise is also caused by the abruptness in the change of the pressure of the gas column or the acceleration of the gas flow. ${ }^{8}$ If the time lag between the exhaust valve opening and the peak pressure is extremely short, the sudden explosive mixture shoots down the exhaust pipe which is capable of setting up a severe sound wave when reaching the atmosphere. ${ }^{9}$ The waves may be audible or inaudible. Those, which are of such low frequency that cannot be heard, can often be felt in the form of mechanical vibration. ${ }^{10}$

\section{Principle of Noise Suppression \\ Control at the Source}

The combustion noise can be reduced at source by suitable design of combustion chambers and by the alteration of the injection timing and fuel spray characteristics. ${ }^{10}$ By proper design of the machine or the system in such a way that the minimum noise is produced or by arresting its propagation at the source itself, the noise can be reduced. However this is not feasible. ${ }^{11}$

When the natural frequency of the engine structural elements coincides with the firing frequency, the resonant vibration is set up and consequently greater noise is generated. This can be suppressed by isolating the noise-radiating surface from the engine, by reducing the area of the radiating surface or by alternating the stiffness of the structure. ${ }^{11}$

\section{Control of Transmission Path}

This is most useful method in such case where the noise reduction at the source is not possible. This may be done as follows:

a. Path deflection: The noise is made to follow a deflected path. Barriers in open air can be effective when they are large in size compared with wavelength of the noise to be deflected. Deflectors can be so arranged that noise be deflected away from the receiver. ${ }^{12}$

b. Enclosure: Considerable attenuation can be obtained by enclosing the source of receiver by properly designed enclosure. This type of noise reduction is made use of suppressing the noise 
produced by heavy diesel engines in the power plants. ${ }^{13}$

c. Absorption: This is done by interposing some material such as rubber, felt, fibre, foil, etc., which have poorer or dissimilar transmission characteristics in the path of the noise. ${ }^{14}$

d. Impedance mismatch: The flow of acoustic energy along the path from the source to the receiver can be impended by discontinuities, which reflect the energy back to the source. Acoustic filters and mufflers operate on this principle, although some mufflers may also include absorption in the transmission path. ${ }^{15}$

\section{The use of Preventive Measures at the Receiver}

Some preventive measures like sound absorbing material or a sound insulating material can be placed to avoid the effect of noise.

\section{Exhaust Noise Suppression}

The exhaust noise reduction can be achieved by the following methods:

i. By converting the high pressure energy into kinetic energy which is then dissipated as heat by turbulence and friction e.g. Motor cycle silencing. ${ }^{16}$

ii. Dissipation: The exhaust is either made to pass through some absorptive material or throttled. But this type of reduction offers more resistance to the flow of exhaust gases which is not desirable. ${ }^{17}$

iii. Reactive type: In this case the various chambers and inter-communicating tubes are arranged in series. This type passes all sub-audible frequencies and filters out those which lie above a certain value. So a free flow of exhaust gases is possible without any throttling effect and that is why this type of mufflers finds more application. ${ }^{18}$

iv. Interference type: The exhaust stream is split up into large number of partial streams of different lengths so that the partial sound oscillation when reunited is mutually displaced in phase. ${ }^{17}$ Oscillations that are $180^{\circ}$ out of phase are cancelled out but not any other oscillations. But this type is not much useful for the engines, which have to be operated under variable speed conditions. ${ }^{18}$

\section{Types of Particulate Traps}

\section{(a) Wire Mesh Particulate Trap}

The trap substrate is made from a knitted stainless steel wire mesh compressed between perforated cylindrical inner and outer casing. The wire mesh is woven and rolled to give a low density and surface to volume ratio on the outer region of the annular shaped substrate, with an increasing density and surface to volume ratio towards the inner diameter region. ${ }^{19}$ Thus the larger particulates are trapped in the outer circumferential mesh whereas the smaller particulates are obstructed towards the inner diameter. This progressive change from an open outer diameter mesh to a more closed inner diameter mesh allows different sized particulates to be trapped at various depths. ${ }^{20}$ It therefore prevents all the particulates collecting only in the outer region of the mesh, where they could quickly clog the filter media. A refractory wash coat is deposited onto the wire mesh, which is then speckled with the noble metals as the catalyst. The wire mesh gives the gas a high degree of turbulent exhaust flow, which produces an effective particulate trap and oxidizer. ${ }^{19}$

\section{(b) Silica Fibre Candle Particulate Trap}

The candle particulate trap incorporates nine filtering elements consisting of punched sheet metal support tubes with one end closed and around these tubes are woven layers of silicon fibre yarn in a cross wire pattern to form the filtration media. ${ }^{21}$ Because these elements resemble candle sticks, they are known as candle elements. The silicon- di- oxide yarn thread has a diameter between 0.7 to $1.0 \mathrm{~mm}$, which in turn is made up of individual fibres of about $9 \times 10^{-6}$. Depositing a refractory wash coat over the woven yarn and impregnating its exposed surface with precious metal can achieve catalytic oxidation. This trap oxidizer produces a very low exhaust gas backpressure and it is subjected to only low thermal and mechanical stresses. But it suffers from a low particulate loading capacity and thus requires frequent regeneration. ${ }^{22}$ 


\section{(c) Ceramic Foam Trap}

The trap consists of ceramic foam made from alumina or cordierite. They exhibit a fairly narrow distribution of wide and virtually circular pores, $250-500 \mu \mathrm{m}$, winding in depth, with few non-through channels. ${ }^{20}$ These filters appear to be more suitable for large flows. They offer low pressure drops and exploit the impaction trapping principle to reduce the pressure drops even further and improve the distribution of the mass of soot collected, no through channels in the cylindrical volume of ceramic foam. This structure ensures more uniform propagation of heat flame during regeneration, lowering the ignition temperature by about $50^{\circ} \mathrm{C}$. The trap is manufactured by impregnating a polyurethane foam matrix with a ceramic paste. ${ }^{19}$

\section{(d) Porous ceramic honeycomb particulate trap}

The oxidizer consists of a porous cellular ceramic honeycomb structure, which has parallel passages of approximately circular section. Alternative passage ways are blocked at either end so that inlet and exit faces resemble the black and white square of a chessboard. The inlet passageways are plugged at far outlet end; whereas the exit passage way is plugged at the inlet ends and are open at the outlet. The exhaust gas entering the inlet passage is compelled to pass at right angles through the walls of the porous ceramic into the exit passageways and finally the filtered exhaust gas is expelled to rear outlet chamber of the particulate trap. Particulate removal from the honeycomb trap is affected by the pressure drop across the filter and as the passageways become partially clogged, this necessitates more fuel to be injected to make up for the resulting power loss. Consequently more heat energy passes out with the exhaust gas and it therefore raises the gas temperature, which activates the ignition and oxidation of the accumulated particulate matter. ${ }^{21}$

In the preceding paragraph, the various types of trap systems used as devices for the after treatment of diesel engine exhaust gas are given. The cost of the porous ceramic honeycomb filter is cheap and it is more efficient than other types of oxidizers, because the porous network structures have low mass, low density and low thermal conductivity. Permeability is also high in this case along with strength, resistance to temperature and structural uniformity is more. Hence, it is proposed to use the ceramic honeycomb filter type trap oxidizer in the present investigation. The objective of this study is to reduce noise emissions from an engine using a particulate trap and a muffler and to compare the reduction in noise levels. ${ }^{22}$

\section{Fabrication of Particulate Trap Fabrication of Outer Casing}

A $540 \mathrm{~mm}$ X $710 \mathrm{~mm}$ X $3 \mathrm{~mm}$ mild steel sheet is taken and polished on the surface. Then it is rolled and welded longitudinally to form a cylindrical outer shell of diameter $170 \mathrm{~mm}$. The welded portion is cleaned and polished. The inner surface of the outer casing is coated with ceramic, which is high thermal resistant, corrosion resistant and sound insulator to avoid the reflection of sound and for absorbing the exhaust noise. ${ }^{6}$ Thus the outer casing of the required dimensions is fabricated and is shown in the Fig. -1 .

\section{Fabrication of Inner core}

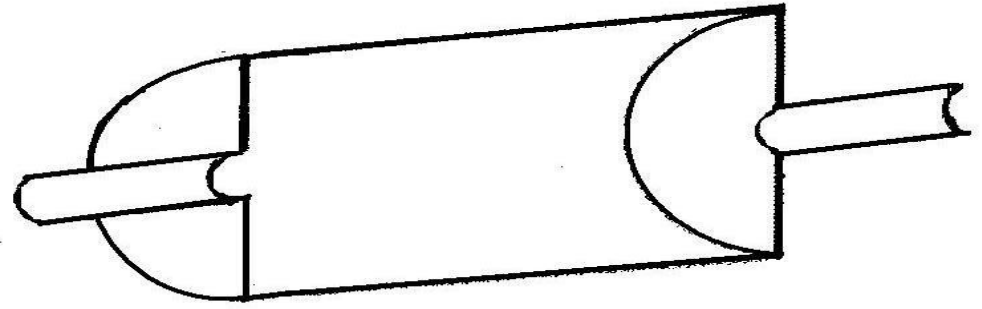

Fig.-1: Trap Outer Casing

A perforated sheet of dimension $79 \mathrm{~mm} \mathrm{X} 300 \mathrm{~mm}$ with hole diameter $6 \mathrm{~mm}$ is rolled to form a perforated tube of diameter $25 \mathrm{~mm}$. The welded portion is cleaned and polished. Twenty-four such perforated tubes are produced. A circular sheet of diameter $170 \mathrm{~mm}$ and thickness $3 \mathrm{~mm}$ is taken and twenty-four uniform 
holes of diameter $25 \mathrm{~mm}$ are drilled in a zigzag manner. Then, for each hole, a perforated tube is welded, the process being repeated for all the remaining tubes. On the other end, a plain circular sheet of diameter $170 \mathrm{~mm}$ and thickness $3 \mathrm{~mm}$ is welded, thus the other ends of the perforated tubes are blocked. A few holes are drilled on the remaining portion of the plain circular sheet through which the exhaust gas will escape. $^{7}$

The inner core thus prepared is coated with the selected catalyst on the entire inner and outer surfaces. Thus the inner core is prepared and is shown in the Figure-2.

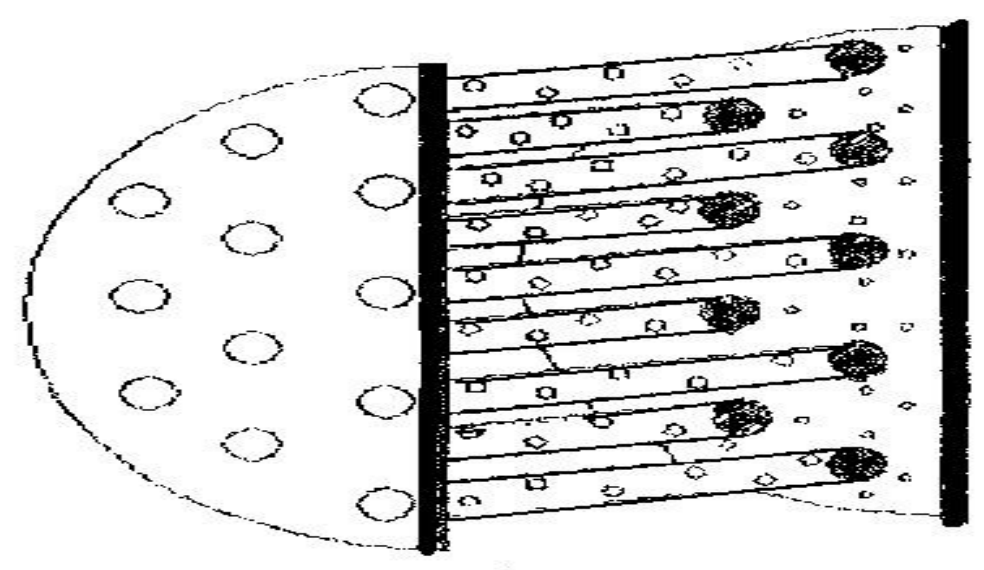

Fig.-2: Sectional view of the trap inner core

\section{Assembling of Inner Core and Outer Casing}

The inner core is then placed inside the outer casing leaving a gap of $20 \mathrm{~cm}$ on both sides. Then the circumference of the circular sheets is welded to the inner surface of the outer casing.

Two circular sheets of the same diameter of $170 \mathrm{~mm}$ are taken and a central hole of diameter $60 \mathrm{~mm}$ is drilled. It is then welded along the circumference to the outer casing.

Two plain tubes of diameter $60 \mathrm{~mm}$ are taken and welded to the end plates of the outer casing along the holes to provide inlet and outlet passages. ${ }^{16}$

\section{EXPERIMENTAL}

The experimental set-up is as shown in the Figure-3. The engine used in this investigation is a four stroke, water cooled, single cylinder, direct injection, and vertical diesel engine at $3.78 \mathrm{KW}$ running at $2000 \mathrm{rpm}$. The engine is mounted on the bed with suitable connections for lubrication and cooling water supply. The fuel is supplied from a fuel tank with three way cock to allow the fuel either from the tank or through the burette. ${ }^{12}$

\section{Exhaust Temperature}

\section{RESULTS AND DISCUSSION}

Figure-4 shows the variation of exhaust gas temperature with the brake power of the engine with and without particulate trap and muffler. Upto $20 \%$ load, the exhaust temperature increases slowly. Beyond $20 \%$ load, the exhaust temperature increases rapidly. ${ }^{11}$

It can be observed that when the engine is fitted with a particulate trap the increase in the exhaust temperature is lower at low loads and at high loads the increase in exhaust gas temperature is higher. For the case with muffler, there is a slight reduction in exhaust gas temperature. The reduction is lesser at low loads and increases slightly with higher loads. ${ }^{9}$

The exhaust temperature of the engine increases by about $10 \%$ at maximum load and by about $5 \%$ at low load when it is fitted with the trap. For the muffler the exhaust temperature decreases by about $2 \%$ at low load and by about $3 \%$ at maximum load. ${ }^{7}$ 
RASĀYAN $J$. Chem.

Vol. 10 | No. 2 |434 - 441 | April - June I 2017

THERMOCQUPLES

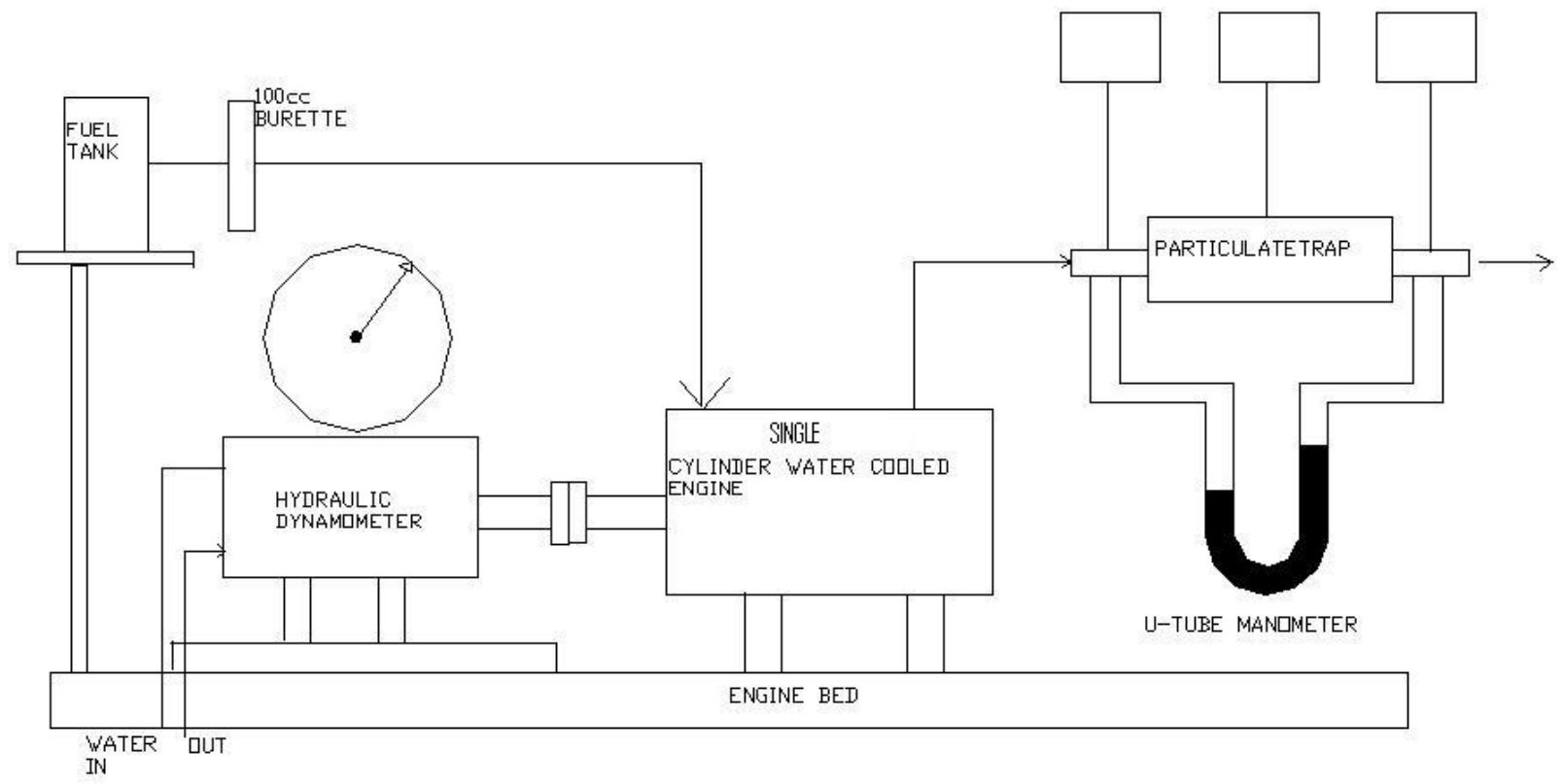

Fig.-3: Experimental setup for Noise Measurement

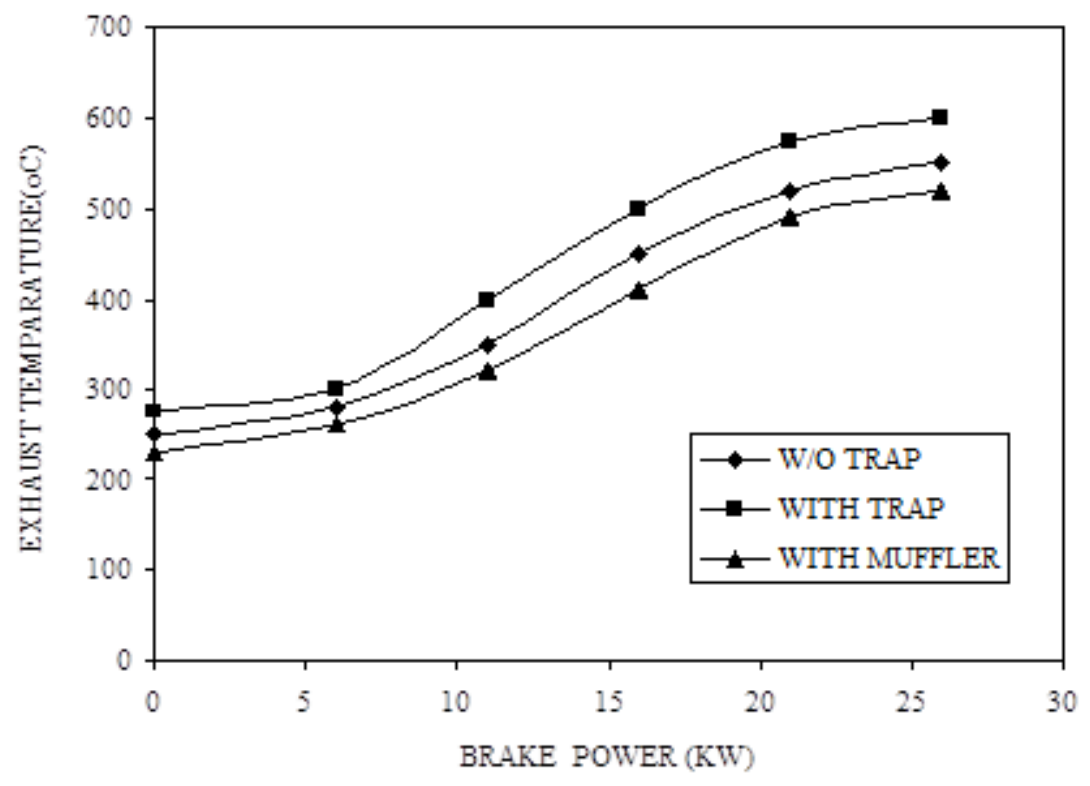

Fig.-4: Variation of exhaust temperature with brake power

\section{Brake Thermal Efficiency}

Figure-5 shows the variation of brake thermal efficiency with respect to brake power. The graph shows that brake thermal efficiency of the engine is increasing as the load increases. Upto $40 \%$ of the load, the brake thermal efficiency is increasing rapidly and beyond that it is increasing gradually. ${ }^{17}$

It is noted that for the engine fitted with particulate trap, the brake thermal efficiency is lesser than for the engine without trap, which is due to the increase in fuel consumption of the engine with trap. The brake thermal efficiency of the engine reduces by about $8 \%$ at maximum load when it is fitted with trap and by about $3 \%$ when it is fitted with muffler. ${ }^{18}$ 


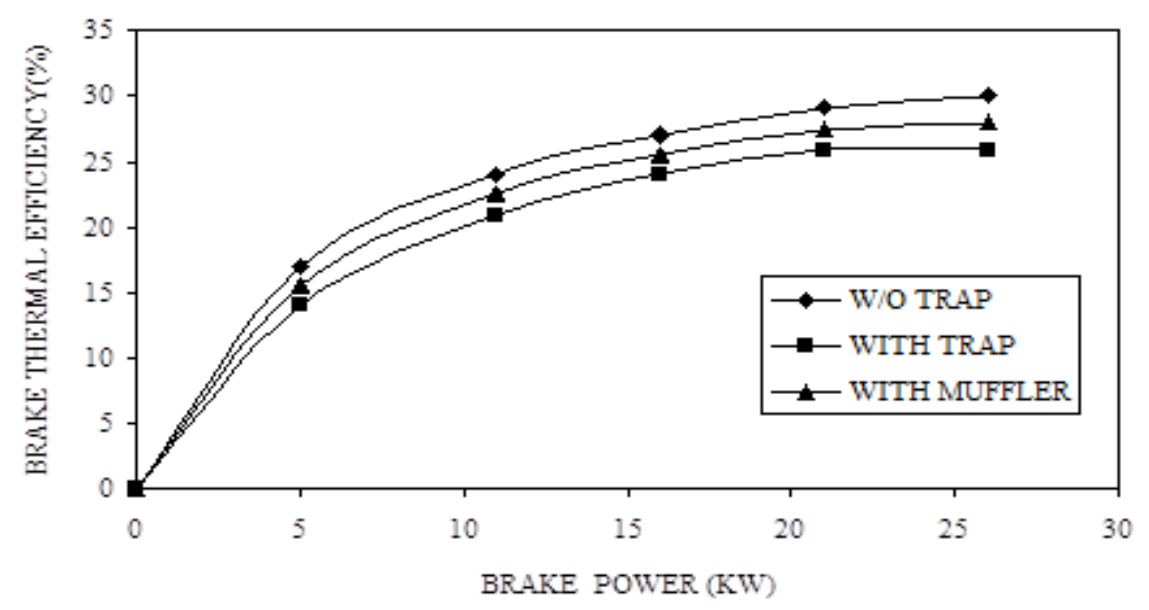

Fig.-5: Variation of brake thermal efficiency with brake power

\section{REFERENCES}

1. H. Schweitzer and A. Mucklow, ASME Paper, 74, (1952).

2. Takeshiabe, Keisuke Yoshimori, and Akiraf Azuma, SAE, Paper 850326.

3. Atsuo Shimura, SAE, Paper 852266.

4. C. Peter, C. Lai and Warner Seodel, SAE, Paper 980281.

5. Robert J. Bernhard and Raw W. Herrick, SAE, Paper 850791.

6. Francisco Payri, Antoni J. Torregrose, and Alberto Broatch, SAE, Paper. 980282.

7. Roy Douglas and Brendan P. Carberry, SAE, Paper 961807

8. J.D. Duke, and V.K. Vadar, SAE, Paper 981415.

9. Avinash R. Patil and P. R. Sajanpawar, SAE, Paper 960189.

10. Marty A. Barris and Eugene D. Wilson. James Hough. W, SAE, Paper 920369.

11. Zhang Guanglong. G, Liang Lunhui, Chen Jiahua and Zhao Xiuren, SAE, Paper 941773

12. Shosuke Oku and Yugoro Masuda, Kaneoba Ltd., SAE, Paper 960192.

13. J. Kellenbach and U. Zink, Corning, SAE, Paper 973049.

14. Arun D. Jatkar, SAE, Paper 971032.

15. Ichiro Sagamota and Takeharu Tanaka, SAE, Paper 970500.

16. R. Sundara Raman, G. Sankaranarayanan and N. Manoharan, International Journal of Applied Engineering Research, 9(18), 3949 (2014).

17. R. Sundara Raman, G. Sankaranarayanan and N. Manoharan, International Journal of Applied Engineering Research, 9(18), 3939 (2014).

18. R. Sundara Raman, G. Sankaranarayanan and N. Manoharan, International Journal of Mechanical Engineering and Technology, 6(10), 66 (2015).

19. R. Sundara Raman, G. Sankaranarayanan and N. Manoharan, International Journal of Mechanical Engineering and Technology, 6(10), 78 (2015).

20. R. Sundara Raman, G. Sankaranarayanan and N. Manoharan, International Journal of Mechanical Engineering and Technology, 6(10), 94 (2015).

21. S. Sendilvelan and K. Bhaskar, Rasayan Journal of Chemistry, 9(4), 692 (2016).

22. S. Sendilvelan and K. Rajan, Rasayan Journal of Chemistry, 10(1), 190 (2017).

[RJC-1629/2017] 